\title{
Probing Electronic Structure of Biomineralized Hydroxyapatite inside Nanoclay Galleries
}

\author{
Scott A. Payne, Dinesh R. Katti, Kalpana S. Katti*, \\ Department of Civil and Environmental Engineering, North Dakota State \\ University, Fargo ND 58105, USA
}

*Corresponding author: Kalpana.Katti@ndsu.edu, Ph: 701-231-9504, Fax: 701-2316185 


\begin{abstract}
Hydroxyapatite, the most abundant mineral in the human body, is also an important component in design of biomaterials for bone tissue regeneration. Synthetic hydroxyapatite mineralized in the laboratory often does not exhibit the same biological and morphological properties of biogenic hydroxyapatite in human bone. A biomimetic hydroxyapatite structure is synthesized using biomineralization routes inside the clay galleries of montmorillonite clay. Amino acids are used to modify the clay galleries. These amino acids are used to mineralize hydroxyapatite. The molecular interactions between nanoclay, modifiers inside nanoclay (amino acids) and biomineralized hydroxyapatite result in unique morphology, structure and stoichiometry of the biomineralized hydroxyapatite. Prior studies have indicated that this biomineralized hydroxyapatite inside nanoclay galleries is an effective component of tissue engineering bone scaffolds that elicits an optimal biological response from human mesenchymal stem cells. Here, a detailed electron energy-loss spectroscopy (EELS) study is reported that elucidates the differences in hydroxyapatite, biomineralized hydroxyapatite and $\beta$-tricalcium phosphate $(\beta$ TCP). Comparison of EELS low-loss transitions and energy loss near-edge structure (ELNES) of $\mathrm{P}-\mathrm{L}_{2,3}$ edges for these three compounds is done to determine if there are differences in their electronic structures. These changes observed experimentally are compared with prior predictions and simulations using molecular dynamics studies. The simulations predict attractive and repulsive interactions between phosphate, modified MMT clay and aminovaleric acid (amino acid) molecules. Kramers-Kronig analysis is performed on the loss spectra obtained to yield the real and imaginary parts of the dielectric function of the apatites $\left(\varepsilon_{1}\right.$ and $\left.\varepsilon_{2}\right)$. We have also used the $\varepsilon_{2}$ spectra obtained to calculate the AC conductivity spectra for the apatites. This study represents a unique experimental probe into molecular interactions in complex biomineralized
\end{abstract}


hydroxyapatite structures. The small changes observed in the energy loss spectra appear to play important biological roles in biomineralized hydroxyapatite such as the ability to differentiate human mesenchymal stem cells into osteoblasts without growth media.

Keywords: hydroxyapatite, biomimetic, EELS, ELNES, montmorillonite

\section{Introduction}

Through a complex process called biomineralization, living organisms form mineralized tissues for structural support and protection. These mineralized tissues can be considered a nanocomposite consisting of a mineral and an organic phase. Bone is one such mineralized tissue, comprising hydroxyapatite $\left(\mathrm{Ca}_{10}\left(\mathrm{PO}_{4}\right)_{6}(\mathrm{OH})_{2}\right)$ (HAP) and collagen. The need for replacement or repair of damaged bone has given researchers reason to investigate HAP and polymeric nanocomposites as biomaterials for tissue regeneration in bone tissue engineering.

A novel mineralization route of HAP in montmorillonite (MMT) clay galleries modified with 5-aminovaleric acid has been reported (Ambre et al., 2011a). Characterization of the 5aminovaleric-acid-modified MMT clay with mineralized HAP (in-situ HAPclay) by Fourier transform infra-red spectroscopy (FTIR) indicated formation of HAP within the modified MMT clay galleries. Further, x-ray diffraction (XRD) demonstrated that the lattice structure of the HAP in modified MMT clay differed from that of synthetically made HAP. The in-situ HAPclay system was then incorporated into chitosan/polygalacturonic acid (ChiPgA) scaffolds and films

for bone-tissue engineering studies (Ambre et al., 2013). When human mesenchymal stem cells (MSCs) were seeded on these ChiPgA/in-situ HAPclay scaffolds and films, they produced 
mineralized nodules even without the osteogenic supplements normally needed for differentiation to osteoblasts. The ChiPgA/in-situ HAPclay nanocomposite elicits an osteoconductive and osteoinductive response from MSCs, indicating that this biomaterial system is promising for bone-tissue engineering applications. In a related study, when polycaprolactone (PCL)/in-situ HAPclay composite films were seeded with MSCs, scanning electron microscopy (SEM) showed formation of matrix vesicles (Katti et al., 2015b). Subsequent high-resolution transmission electron microscopy (TEM) studies showed vesicles containing highly crystalline minerals indicating that this system is favorable for bone mineral formation. Molecular dynamic (MD) simulations were carried out on the HAP/5-aminovaleric acid/MMT system to determine the extent of the molecular interactions among the different components (Katti et al., 2015a). High attractive and high repulsive interactions were found between $\mathrm{PO}_{4}{ }^{3-}$ and MMT clay. The large non-bonded interaction in the in-situ HAPclay indicates the influence of the neighboring environment on $\mathrm{PO}_{4}{ }^{3-}$. These computational studies provide a framework for materials design and selection for biomaterials to be used in tissue engineering.

Identification of mineral composition is challenging in elucidating biomineralization processes because mineralization occurs on a level requiring instrumentation with nanometerscale spatial resolution. The only technique available to directly provide information about the chemical and coordination environment of minerals at nm scale spatial resolution is TEM-based electron energy-loss spectroscopy (EELS). Transmission electron microscopy provides high spatial resolution while allowing visual correlation to diffraction and elemental-composition data. Electron energy-loss spectroscopy is a sensitive technique for probing electronic structure at the molecular level for a variety of materials (Brockt and Lakner, 2000; Katti et al., 2002; Murdoch et al., 2014; Virdi et al., 2013). The EELS spectrum, created by electrostatic 
interactions of the incident electron with the sample electrons, consists of three unique regions: zero loss peak (ZLP), low loss $(0-50 \mathrm{eV})$, and core loss $(>50 \mathrm{eV})$. Egerton (2011) provides a detailed description of the electron scattering phenomenon and applications to materials characterization. The ZLP represents elastically scattered electrons that have undergone immeasurably small or no energy loss, used as a reference $(0 \mathrm{eV})$, and is the most dominant feature of the EELS spectrum. The width of the ZLP generally $0.2-2 \mathrm{eV}$, is related to energy distribution of the emission source of the TEM. The low-loss region $(0-50 \mathrm{eV})$ represents the interaction of the incident electrons with the outer shell (valence) electrons of the sample atoms. The dominant feature of the low-loss region, resulting from excitation of the collective resonances of valence electrons, is called a plasma resonance and it takes the form of a longitudinal traveling wave (Egerton, 2011) Features in the core-loss region $(>50 \mathrm{eV})$ of the EEL spectrum are called ionization edges. These arise from the excitation of a core electron into the unoccupied electron states through interaction with the incident electron beam. Ionization edges are located at an energy loss value equivalent to the critical ionization of that particular core electron from a specific element. The relative edge intensity can be used to evaluate material composition quantitatively. Fine spectral details routinely found $0-50 \mathrm{eV}$ beyond the ionization edge are called electron loss near-edge structure (ELNES) and reflect the energy distribution of those unoccupied states (unoccupied density of states, DOS). Electron loss nearedge structure depends on the local atomic environment such as element-specific coordination, valence, and anisotropic density of states, which are used to elucidate electronic structure of materials. Analytical TEM-EELS has been used to investigate synthetic HAP systems and some types of mineralized tissue, e.g., natural apatite (Gregori et al., 2006; Liou et al., 2004) and carbonate from minerals of biological origin (Kłosowski et al., 2015). However, low-loss EELS 
with detailed interpretation of corresponding edges has seldom been employed. High angular annular dark field/TEM-EELS was used to investigate mineral-collagen interactions in elephantivory dentin, which has a structure similar to bone, but detailed interpretation of the corresponding edges was not attempted (Jantou-Morris et al., 2010). Human enamel, peritubular dentin and intertubular dentin have been characterized using EELS/ELNES (Srot et al., 2012) and near-edge structures have been compared for synthetic and biological bone specimens containing an anti-osteoporotic drug (Rossi et al., 2014).

Because of the importance of these materials for bioengineering applications, TEM-EELS was used to evaluate differences and similarities among HAP, biomimetic in-situ HAPclay, modified MMT clay and $\beta$-TCP. In particular, EELS low-loss transitions and ELNES of P- $\mathrm{L}_{2,3}$ edges were compared to determine if there are electronic structural differences. Here we report detailed experimental analysis of electron energy-loss spectra from synthetic HAP, biomimetic HAP created using biomineralization routes inside clay galleries (in-situ HAP clay), MMT clay and $\beta$-TCP systems.

\section{MATERIALS AND METHODS}

\subsection{Materials}

Sodium montmorillonite (Na-MMT) clay (SWy-2, Crook County, Wyoming USA) with a cation exchange capacity of $76.4 \mathrm{meq} / 100 \mathrm{~g}$ was obtained from the Clay Minerals Repository at the University of Missouri, Columbia. The modifier 5-aminovaleric acid and $\beta$-TCP were purchased from Sigma-Aldrich. For the preparation of HAP and in-situ HAPclay, sodium phosphate $\left(\mathrm{Na}_{2} \mathrm{HPO}_{4}\right)$ was supplied by JT Baker and calcium chloride $\left(\mathrm{CaCl}_{2}\right)$ by Electron 
Microscopy Sciences. Lacey carbon on 300 mesh copper grids were purchased from Ted Pella, Inc.

\subsection{Methods}

Hydroxyapatite, modified MMT clay, and in-situ HAPclay previously prepared for use in other studies (Ambre et al., 2011) were used for the current investigations. Preparation of these materials has been described in detail elsewhere ( $\{$ Katti, 2010, Use of unnatural amino acids for design of novel organomodified clays as components of nanocomposite biomaterials $\}$ Katti et al. 2010; Ambre et al., 2011; Ambre et al, 2013; Ambre et al. 2015) and is summarized in the following sections.

\subsubsection{Preparation of $H A P$}

Hydroxyapatite was prepared using the wet precipitation method (Verma et al., 2006): a $39.8 \mathrm{mmol}$ solution of $\mathrm{CaCl}_{2}$ was added dropwise to a $23.8 \mathrm{mmol} \mathrm{NaHPO}_{4}$ solution in distilled water and stirred. The resulting slurry was centrifuged, decanted, washed with distilled water, dried, and sieved to produce HAP powder.

\subsubsection{Preparation of modified MMT clay}

To prepare modified MMT clay, Na-MMT clay was suspended in distilled water heated to $60^{\circ} \mathrm{C}$. While stirring, 5 -aminovaleric acid was added and the solution was stirred at $60^{\circ} \mathrm{C}$ for 1 hour. The resulting mixture was centrifuged and the liquid decanted. The remaining slurry was washed with distilled water, centrifuged, decanted, dried at $70^{\circ} \mathrm{C}$, and sieved to yield modified MMT clay powder.

\subsubsection{Preparation of in-situ HAPclay}


Modified MMT clay was stirred with a $23.8 \mathrm{mmol}$ solution of $\mathrm{NaHPO}_{4}$ for 2 hours to ensure dispersion. A $39.8 \mathrm{mmol}$ solution of $\mathrm{CaCl}_{2}$ in distilled water was then added dropwise to the suspension and stirred for 8 hours while maintaining a $\mathrm{pH}$ of 7.4 by adding $\mathrm{NaOH}$. The solution was allowed to settle for 24 hours and the liquid was decanted. The precipitate was centrifuged to remove excess water and dried at $70^{\circ} \mathrm{C}$. The dried precipitate was then sieved and ground to yield in-situ HAPclay.

\subsection{Sample preparation for TEM-EELS experiments}

Small amounts of HAP, in-situ HAPclay, $\beta$-TCP, and modified MMT clay were placed in separate test tubes. One to two milliliters of absolute ethanol was added to each and the test tubes were placed in a sonication bath for five minutes to disperse the particles. Approximately one microliter of each sample was transferred to a 300-mesh copper TEM grid with a lacey carbon support film. Torn filter paper was used to remove the excess liquid and the grids were air-dried prior to characterization by TEM-EELS.

\subsection{TEM-EELS characterization}

Brightfield images and EELS data from each sample were acquired using a JEOL JEM$2100 \mathrm{LaB}_{6}$ TEM equipped with a Gatan Orius SC1000 CCD camera and a Gatan ENFINA 1000 electron energy loss spectrometer. All EELS spectra were recorded in diffraction mode with a 10 $\mathrm{nm}$ probe size. The convergence angle $(\alpha)$ was $12.53 \mathrm{mrad}$ and the collection semi-angle $(\beta)$ was $14.43 \mathrm{mrad}$. Low-loss and core-loss spectra were collected using a $1 \mathrm{~mm}$ entrance aperture and a $0.1 \mathrm{eV} /$ channel dispersion. All spectral data processing was completed using Gatan Microscopy Suite with DigitalMicrograph Software (DM) (ver. 1.85 1535). Background contribution of the ZLP signal was removed from each core-loss spectrum by subtraction of a 
power-law fit to a window approximately $10 \mathrm{eV}$ wide before the $\mathrm{P}-\mathrm{L}_{2,3}$ edge. The $\mathrm{x}$-axis window was situated so the background-subtracted spectrum did not intersect the energy-loss axis in the DM preview. Deconvolution of each $\mathrm{P}-\mathrm{L}_{2,3}$ core loss spectrum was accomplished using the Fourier-ratio method to remove any effects of plural scattering. The $\mathrm{P}-\mathrm{L}_{2,3}$ core loss spectra then were normalized to the intensity of the $138 \mathrm{eV}$ peak and smoothed using a Savitzky-Golay function. Background subtraction and deconvolution of each low-loss spectrum was done in a single step using the Fourier-log method. Kramers-Kronig analysis was used to calculate the $\varepsilon_{1}$ and $\varepsilon_{2}$ plots and energy loss function (ELF) from each deconvolved low-loss spectrum using a refractive index of 1.63 with 100 iterations. The low-loss spectra were normalized for intensity using the first major peak ( $10 \mathrm{eV}$ for $\varepsilon_{1}$ and $\varepsilon_{2}, \sim 20 \mathrm{eV}$ for ELF) and smoothed using a Savitzky-Golay function. Energy positions for the peaks were determined by superimposing the calculated second derivative spectrum onto the original spectrum.

\section{Results and discussion}

Crystal structures of hydroxyapatite, $\beta$ TCP, organically modified clay and also clay-HAP are shown in Fig. 1. Representative TEM images of in-situ HAPclay, HAP, $\beta$-TCP, and modified MMT clay are shown in Fig. 2. The low-magnification image of in-situ HAPclay (Fig. 1a) shows clusters of grains. The higher-magnification image (Fig. 2b) reveals the lattice structure and biomimetic rounded shapes interspersed within the clay sheets. At low magnification, HAP (Fig. 2c) appears similar to in-situ HAPclay (Fig. 2a); however, the rounded structures are not present

(Fig. 2d). Elongated needle-like crystals of $\beta$-TCP (Fig. 2e) show lattice structure parallel to their long axis at higher magnification (Fig. 2f). Modified MMT clay is entirely different from the 
other samples, with its agglomerated individual clay sheets (Fig. 2g); at higher magnification, the lattice pattern of the clay sheets is apparent (Fig. 2h).

\subsection{Low-loss spectra}

Electron energy-loss spectroscopy analysis was performed on $\beta$-TCP, synthetic HAP, in-situ HAPclay, and modified MMT clay. Two energy-loss ranges were chosen for analysis: (I) the low-loss region from 0-65 eV containing the $\mathrm{Ca}-\mathrm{M}_{2,3}$ edge (Figs. 3-4) and (II) the core-loss region from 130-210 eV including the $\mathrm{P}-\mathrm{L}_{1}$ and $\mathrm{P}-\mathrm{L}_{2,3}$ ionization edges (Fig. 5).

Deconvolution by the Fourier-log method subtracted the ZLP and removed contributions from plural scattering for each of the low-loss spectra. The resulting energy loss function (ELF) plots for $\beta$-TCP, HAP, and in-situ HAPclay represent single scattering distribution (SSD) functions (Fig. 3).

These spectra consistently show two main peaks located at approximately $23 \mathrm{eV}$ and 36 $\mathrm{eV}$, labeled A and B respectively. Modified MMT clay spectra show a single peak at $23 \mathrm{eV}$. Corresponding peak positions from the energy loss function plots in Fig. 3 are summarized in Table 1.

Peak $A$ is attributed to the bulk plasmon as determined through $\varepsilon_{1}-\varepsilon_{2}$ comparison (Fig. 4). A weak feature $\left(\mathrm{B}^{*}\right)$ around $28 \mathrm{eV}$ is present between peaks $\mathrm{A}$ and $\mathrm{B}$. Peaks $\mathrm{B}$ and $\mathrm{B}^{*}$ correspond to the Ca-M $\mathrm{M}_{2,3}$ ionization edge (Ahn and Krivanek, 1983). The modified MMT clay spectrum does not exhibit the peaks $\mathrm{B}$ and $\mathrm{B}^{*}$ because calcium is absent. Low-loss spectra among samples differ in the presence or absence of fine structure on the low-energy side of peak A. Table 2 summarizes peak shifts and peak A:B ratios in the ELF.

Since peak B intensity varies, the A/B peak ratio of the in-situ HAPclay spectrum is close to one, while A/B peak ratios of HAP and $\beta$-TCP are less than one. Peak B of in-situ HAPclay is 
shifted $(-0.5 \mathrm{eV})$ from $\beta$-TCP and $(-0.7 \mathrm{eV})$ from HAP. Peak $\mathrm{B}^{*}$ of in-situ HAPclay is shifted $(+1.3 \mathrm{eV})$ from $\beta$-TCP and $(+1.1 \mathrm{eV})$ from HAP. The spectra of $\beta$-TCP and HAP are similar to one another, but they differ markedly from the spectrum of in-situ HAPclay.

\subsection{Kramers-Kronig analysis}

Kramers-Kronig analysis was used to calculate $\varepsilon_{1}$ and $\varepsilon_{2}$ from the SSD spectrum of each sample: $\varepsilon_{1}$ represents collective electron excitations (bulk plasmon) (Fig. 4a); $\varepsilon_{2}$ represents single-electron transitions (Fig. 4b).

The $\varepsilon_{1}$ and $\varepsilon_{2}$ plots for each sample were compared to determine which features of the lowloss spectra were single transitions and which were due to bulk plasmon resonance. The bulk plasmon resides around $23 \mathrm{eV}$. The fine structure of the $\varepsilon_{2}$ plots represents single-electron transitions related to electronic structure (Fig. 4b). Single-electron transitions from the $\varepsilon_{2}$ plots are summarized in Table 3. The modified MMT clay $\varepsilon_{2}$ spectrum shows a major peak at $10.5 \mathrm{eV}$ and a minor peak located at $17.4 \mathrm{eV}$. No fine structure is seen on the low-energy side of the major peak. The $\beta$-TCP $\varepsilon_{2}$ spectrum is dominated by a narrow and very intense transition at 9.4 $\mathrm{eV}$. There is very little fine structure on the low-energy side of this peak, with a weak transition at $3.0 \mathrm{eV}$ being the only discernible feature. The $\varepsilon_{2}$ spectra of HAP and in-situ HAPclay are similar, with $\beta$-TCP transitions around 9.8, 14.8, 20.5, 28.3 and $33.3 \mathrm{eV}$. There is more fine structure on the low-energy side of the peak at $9.8 \mathrm{eV}$ for HAP and in-situ HAPclay than for $\beta$ TCP. This $9.8 \mathrm{eV}$ peak is broadened by the amount of fine structure located on the high-energy side. While there are some overlapping transitions between HAP and in-situ HAPclay (3.9, 30.6 
$\mathrm{eV})$ that do not overlap with $\beta$-TCP, in-situ HAPclay has more unique transitions $(1.0,5.8,13.3$, 17.6, 19.5 and $25.1 \mathrm{eV}$ ) than either of the other materials.

The $\varepsilon_{2}$ plots were used to calculate the high-frequency AC conductivity plots for in-situ HAPclay, HAP, $\beta$-TCP, and modified MMT clay (Fig. 5). As seen, the plots of in-situ HAPclay and HAP are similar to each other and quite different from those for $\beta$-TCP and modified MMT clay.

\subsection{P-L $L_{2,3}$-edge spectra}

The P- $\mathrm{L}_{2,3}$-edge EELS spectra of $\beta$-TCP, HAP, and in-situ HAPclay express features in five energy-loss regions (Fig. 6), characterized by two sharp peaks located at 138-139 eV (A) and 146-147 eV (B), followed by a broader, more intense peak starting around $153 \mathrm{eV}$ (C).

A weaker feature $\left(\mathrm{A}^{*}\right)$ is often located as a shoulder on the high-energy side $(+2-3 \mathrm{eV})$ of peak A. The $\mathrm{P}-\mathrm{L}_{1}$ ionization edge (D) is present around $189 \mathrm{eV}$. The $\mathrm{P}-\mathrm{L}_{2,3}$ edge is represented by two peaks, one at $136 \mathrm{eV}\left(\mathrm{P}-\mathrm{L}_{3}\right)$ and another peak within $\sim 1 \mathrm{eV}\left(\mathrm{P}-\mathrm{L}_{2}\right)$ due to the transitions from spin-orbit split $2 p$ electrons $\left(2 p_{-}\{3 / 2\}\right.$ and $\left.2 p_{-}\{1 / 2\}\right)$ into the first unoccupied $3 \mathrm{~s}-$ antibonding state (Kruse et al., 2009). These peaks, while generally difficult to resolve, are most likely located within the low-energy shoulder of peak A according to synchrotron-based X-ray absorption near-edge structure (XANES) data reported for phosphorus reference compounds (Cosmidis et al., 2015; Kruse et al., 2009). Peak A, located in the 138-139 eV energy-loss region, has been attributed to transitions to the $3 p$-like antibonding state in the Si L-edge spectrum of $\mathrm{SiO}_{2}$, which is isoelectronic with $\mathrm{PO}_{4}{ }^{3-}$ (Harp et al., 1990). These transitions to dipole-forbidden $3 p$ orbitals are made possible by the mixing of characters from other elements 
such as oxygen. Peak $\mathrm{A}^{*}$, located $\sim 142 \mathrm{eV}$ on the high-energy side of peak A, is a broad peak that is unique to calcium phosphates and due to transition of the phosphorus $2 \mathrm{p}$ electron to calcium 3d empty orbitals (Kruse et al., 2009), but also may be due to multiple scattering of the outgoing electron (Sutherland et al., 1993). Peak B, located $\sim 147 \mathrm{eV}$, is known as the d-state resonance or "shape resonance" peak; the energy position of this peak is sensitive to the molecular symmetry and to the local environment of the phosphorus (Kruse et al., 2009; Li et al., 1995). This peak is followed by a broad peak (C) starting at $\sim 150 \mathrm{eV}$ that corresponds to the position of the atomic cross-section maximum for the phosphorus $2 \mathrm{p}$ level relative to its respective $2 \mathrm{p}$ ionization edges (Sutherland et al., 1993; Yeh and Lindau, 1985). The P-L $\mathrm{L}_{2,3}$ core loss region spectral features for $\beta$-TCP, HAP, and in-situ HAPclay as well as transition origins are summarized in Table 4.

Table 5 compares the peak shift values for the sample materials. Peak $A^{*}$, the P $2 p$ to Ca $3 d$ empty orbital transition, shows small differences in energy values among the three samples.

The d-state shape resonance peak, labeled Peak B, shows a shift $(-0.9 \mathrm{eV})$ when comparing in-situ HAPclay to $\beta$-TCP. There is very small change $(-0.3 \mathrm{eV})$ in the Peak B energy position between in-situ HAPclay and HAP. For the 2p level (peak C) in-situ HAPclay and HAP are shifted $+1.0 \mathrm{eV}$ and $+1.2 \mathrm{eV}$ respectively from $\beta$-TCP. The P- $\mathrm{L}_{1}$ peak (D) of in-situ HAPclay and HAP show similar energy values while the $\mathrm{P}-\mathrm{L}_{1}$ peak for $\beta-\mathrm{TCP}$ is shifted -0.4 to $-0.5 \mathrm{eV}$ from in-situ HAPclay/HAP.

\section{Conclusions}

Clearly the HAP biomineralized inside clay galleries, (in-situ HAPclay) is structurally different morphologically from non-biomimetic HAP (Fig. 2). The rounded shapes seen in mineralized 
HAP with amino acid modified nanoclays resemble biogenic HAP in human bone. Shifts in the Ca- $\mathrm{M}_{2,3}$ ionization edge in the in-situ HAPclay and HAP in the ELF are indicative of changes to the Ca outer shell interactions also indicated in prior molecular dynamics simulations that predicted large interaction energies with Ca (Katti et al., 2015a; Sharma et al., 2015). Further, changes to the valence band of the in-situ HAPclay are observed compared to the nonbiomimetic HAP. Experimentally observed changes in the $\varepsilon_{2}$ spectra (Fig. 3) reflect subtle outer shell changes in the electronic structure, as suggested through simulations. These changes result from biomineralization guided by weak non-bonded interactions between $\mathrm{Ca}$ and $\mathrm{P}$ from HAP and amino acid groups inside clay galleries. Further, $\varepsilon_{2}$ was used to calculate the high-frequency AC conductivity plots for in-situ HAPclay, HAP, $\beta$-TCP, and modified MMT clay (Fig. 4.). Again, subtle differences in the valence bands of $\mathrm{Ca}$ and $\mathrm{P}$ in the in-situ HAPclay are observed. The near-edge structure of the P- $\mathrm{L}_{2,3}$ edge was also analyzed. Extremely subtle changes resulting from transitions to the $3 p$-like anti-bonding state, allowable to dipole-forbidden $3 p$ orbitals due to mixed characters from oxygen (Harp et al., 1990) (Table 3). Thus these EELS studies elucidate the small changes that occur in the valence band and outer electronic structures of HAP mineralized inside clay galleries (in-situ HAPclay). Shifts in the Ca- $\mathrm{M}_{2,3}$ ionization edge in the ELF between in-situ HAPClay and HAP indicate Ca outer shell interactions are occurring. The fine structure of the $\varepsilon_{2}$ plots represents single-electron transitions related to electronic structure. The changes observed between HAP and In-situ HAPClay in the $\varepsilon_{2}$ plots reflected subtle outer shell interactions of $\mathrm{Ca}$ and $\mathrm{P}$. The differences result from biomineralization guided by weak non-bonded interactions between $\mathrm{Ca}$ and $\mathrm{P}$ in the in-situ HAPClay from the HAP and amino acid groups inside the clay galleries. This verifies the prediction through molecular dynamics simulations of these interactions (Katti et al., 2015a; Sharma et al., 2015). Molecular dynamics 
simulation in previous work has indicated presence of significant non-bonded interaction between $\mathrm{Ca}$ and $\mathrm{P}$ in the in-situ HAPClay. This illustrates the influence of the neighboring

environment on the $\mathrm{PO}_{4}{ }^{3-}$. The observed changes in the EELS spectra are thus indicative of experimental observation of the changes to the $\mathrm{PO}_{4}{ }^{3-}$ environment.

The use of EELS has shown that small changes occur in the valence band and outer electronic structures of in-situ HAPClay (HAP mineralized within clay galleries) These differences are significant because when biomineralized in-situ HAPClay is incorporated in polymer scaffold systems superior biological functions are demonstrated, such as driving human mesenchymal stem cells towards differentiation into osteoblastic lineages without the use of growth media (Ambre et al., 2013; Ambre et al., 2014).

\section{Acknowledgements}

This material is based upon work supported by the National Science Foundation under Grant No. 0821655 for installation and purchase of electron microscopy equipment. Any opinions, findings, and conclusions or recommendations expressed in this material are those of the author(s) and do not necessarily reflect the views of the National Science Foundation. Support from ND EPSCoR is also acknowledged. Author SAP would like to acknowledge Dr. Jayma Moore for editorial suggestions. manuscript. 


\section{References}

Ahn, C.C., Krivanek, O.L., 1983. EELS Atlas: A Reference Collection of Electron Energy Loss Spectra Covering All Stable Elements. Gatan.

Ambre, A., Katti, K.S., Katti, D.R., 2011. In situ mineralized hydroxyapatite on amino acid modified nanoclays as novel bone biomaterials. Materials Science and Engineering: C 31, 10171029.

Ambre, A.H., Katti, D.R., Katti, K.S., 2013. Nanoclays mediate stem cell differentiation and mineralized ECM formation on biopolymer scaffolds. Journal of Biomedical Materials Research Part A 101, 2644-2660.

Ambre, A.H., Katti, D.R., Katti, K.S., 2014. Biomineralized hydroxyapatite nanoclay composite scaffolds with polycaprolactone for stem cell-based bone tissue engineering. Journal of Biomedical Materials Research Part A, 2077-2101.

Brockt, G., Lakner, H., 2000. Nanoscale EELS analysis of dielectric function and bandgap properties in $\mathrm{GaN}$ and related materials. Micron 31, 435-440.

Cosmidis, J., Benzerara, K., Nassif, N., Tyliszczak, T., Bourdelle, F., 2015. Characterization of Ca-phosphate biological materials by scanning transmission X-ray microscopy (STXM) at the Ca L2,3-, P L2,3- and C K-edges. Acta Biomaterialia 12, 260-269.

Egerton, R., 2011. Electron energy-loss spectroscopy in the electron microscope. Springer Science \& Business Media.

Gregori, G., Kleebe, H.J., Mayr, H., Ziegler, G., 2006. EELS characterisation of beta-tricalcium phosphate and hydroxyapatite. Journal of the European Ceramic Society 26, 1473-1479.

Harp, G.R., Han, Z.L., Tonner, B.P., 1990. X-ray absorption near edge structures of intermediate oxidation states of silicon in silicon oxides during thermal desorption. Journal of Vacuum Science \& Technology A 8, 2566-2569.

Jantou-Morris, V., Horton, M.A., McComb, D.W., 2010. The nano-morphological relationships between apatite crystals and collagen fibrils in ivory dentine. Biomaterials 31, 5275-5286.

Katti, K.S., Ambre, A.H., Peterka, N., Katti, D.R., 2010. Use of unnatural amino acids for design of novel organomodified clays as components of nanocomposite biomaterials. Philosophical Transactions of the Royal Society a-Mathematical Physical and Engineering Sciences 368, 19631980. 
Katti, D.R., Sharma, A., Ambre, A.H., Katti, K.S., 2015a. Molecular interactions in biomineralized hydroxyapatite amino acid modified nanoclay: In silico design of bone biomaterials. Materials Science and Engineering: C 46, 207-217.

Katti, K.S., Ambre, A.H., Payne, S., Katti, D.R., 2015b. Vesicular delivery of crystalline calcium minerals to ECM in biomineralized nanoclay composites. Materials Research Express 2, 045401.

Katti, K.S., Qian, M.X., Dogan, F., Sarikaya, M., 2002. Dopant effect on local dielectric properties in barium titanate based electroceramics determined by transmission EELS. Journal of the American Ceramic Society 85, 2236-2243.

Kruse, J., Leinweber, P., Eckhardt, K.-U., Godlinski, F., Hu, Y., Zuin, L., 2009. Phosphorus L2,3-edge XANES: overview of reference compounds. Journal of Synchrotron Radiation 16, 247-259.

Kłosowski, M.M., Friederichs, R.J., Nichol, R., Antolin, N., Carzaniga, R., Windl, W., Best, S.M., Shefelbine, S.J., McComb, D.W., Porter, A.E., 2015. Probing carbonate in bone forming minerals on the nanometre scale. Acta biomaterialia 20, 129-139.

Li, D., Bancroft, G.M., Fleet, M.E., Feng, X.H., 1995. Silicon K-edge XANES spectra of silicate minerals. Physics and Chemistry of Minerals 22, 115-122.

Liou, S.-C., Chen, S.-Y., Lee, H.-Y., Bow, J.-S., 2004. Structural characterization of nano-sized calcium deficient apatite powders. Biomaterials 25, 189-196.

Murdoch, B., McCulloch, D., Partridge, J., 2014. Structural and dielectric properties of energetically deposited hafnium oxide films. Semiconductor Science and Technology 29, 125014 .

Rossi, A.L., Moldovan, S., Querido, W., Rossi, A., Werckmann, J., Ersen, O., Farina, M., 2014. Effect of strontium ranelate on bone mineral: Analysis of nanoscale compositional changes. Micron 56, 29-36.

Sharma, A., Payne, S., Katti, K., Katti, D., 2015. Evaluating Molecular Interactions in Polycaprolactone-Biomineralized Hydroxyapatite Nanocomposites using Steered Molecular Dynamics. JOM 67, 733-743.

Srot, V., Bussmann, B., Salzberger, U., Koch, C.T., van Aken, P.A., 2012. Linking Microstructure and Nanochemistry in Human Dental Tissues. Microscopy and Microanalysis 18, 509-523.

Sutherland, D., Kasrai, M., Bancroft, G., Liu, Z., Tan, K., 1993. Si L-and K-edge x-rayabsorption near-edge spectroscopy of gas-phase $\mathrm{Si}(\mathrm{CH} 3) \times(\mathrm{OCH} 3) 4-\mathrm{x}$ : Models for solidstate analogs. Physical Review B 48, 14989.

Verma, D., Katti, K., Katti, D., 2006. Bioactivity in in situ hydroxyapatite-polycaprolactone composites. Journal of Biomedical Materials Research Part A 78A, 772-780. 
Virdi, K.S., Kauffmann, Y., Ziegler, C., Ganter, P., Lotsch, B.V., Kaplan, W.D., Blaha, P., Scheu, C., 2013. Electronic structure of KCa 2 Nb 3 O 10 as envisaged by density functional theory and valence electron energy loss spectroscopy. Physical Review B 87, 115108.

Yeh, J.J., Lindau, I., 1985. Atomic subshell photoionization cross sections and asymmetry parameters: $1 \leqslant Z \leqslant 103$. Atomic Data and Nuclear Data Tables 32, 1-155. 


\section{Figure Captions}

Figure 1. Crystal structures of a)Hydroxyapatite b) $\beta$ TCP c) amino acid modified nanoclay and d) modified nanaoclay-HAP

Figure 2. TEM images at low-magnification (left column) and high-magnification (right column). (a,b) in-situ HAPclay, (c,d) HAP, (e,f) $\beta$-TCP, and (g,h) modified MMT clay.

Figure 3. Energy Loss Function spectra of in-situ HAPclay, HAP, $\beta$-TCP, and modified clay. Peak A corresponds to the plasmon peak, and peaks B and $\mathrm{B}^{*}$ correspond to the calcium $\mathrm{M}_{2,3}$ edge. The spectra are separated vertically for clarity and bars denote peak position.

Figure 4. The real $\left(\varepsilon_{1}\right)$ part (a) and imaginary $\left(\varepsilon_{2}\right)$ part (b) of the complex dielectric function obtained from Kramers-Kronig analysis of the EEL spectra for in-situ HAPclay, HAP, $\beta$-TCP, and modified MMT clay The overlaid spectra are separated vertically for clarity.

Figure 5. Calculated high-frequency AC conductivity plots for in-situ HAPclay, HAP, $\beta$-TCP, and modified MMT clay. The overlaid spectra are separated vertically for clarity.

Figure 6. Overlay of the $\mathrm{P}-\mathrm{L}_{2,3}$ core loss region $(135-210 \mathrm{eV})$ obtained from in-situ HAPclay, HAP, and $\beta$-TCP. The spectra are separated vertically for clarity. The peak positions (A, B, C, and D) are denoted by vertical lines and discussed in the text. 
Figure 1
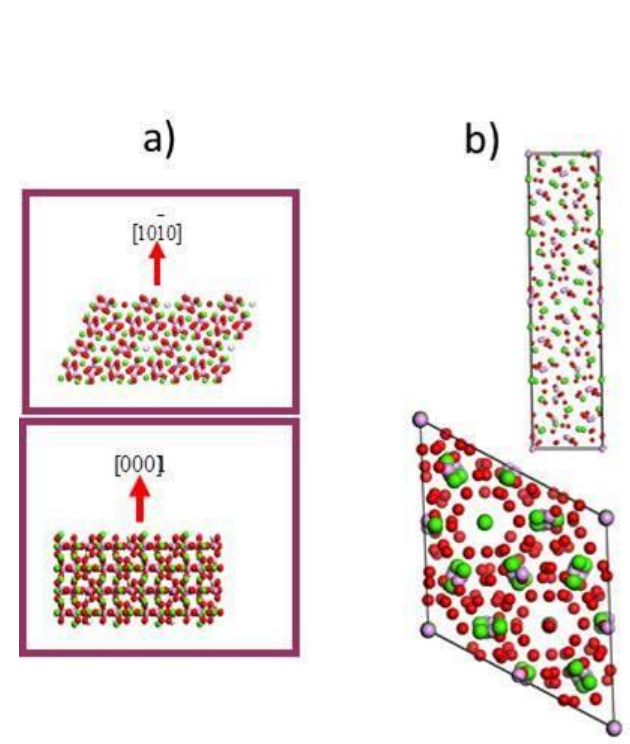

c)

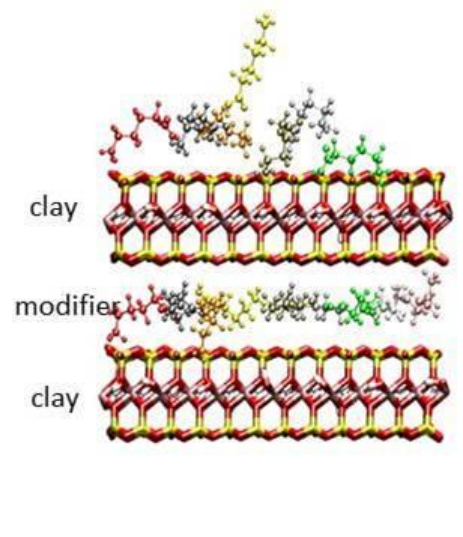

d)

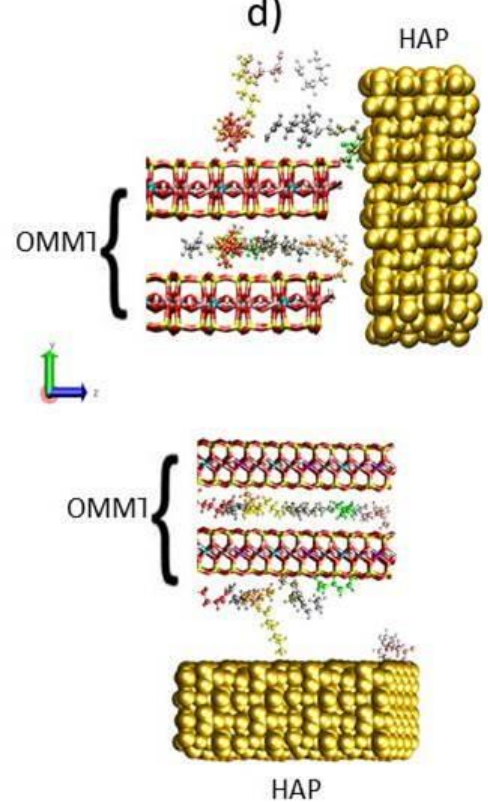


Figure 2
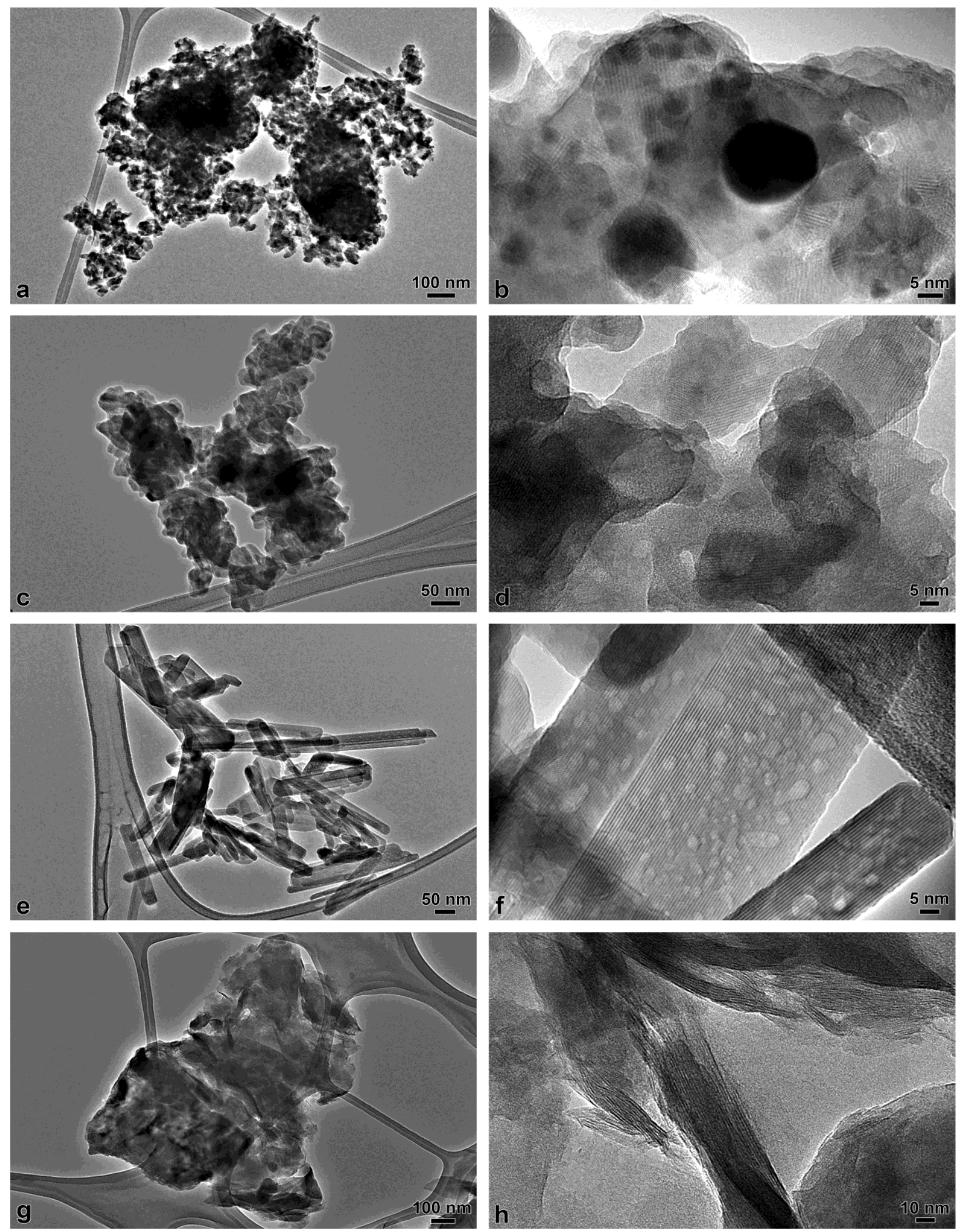
Figure 3

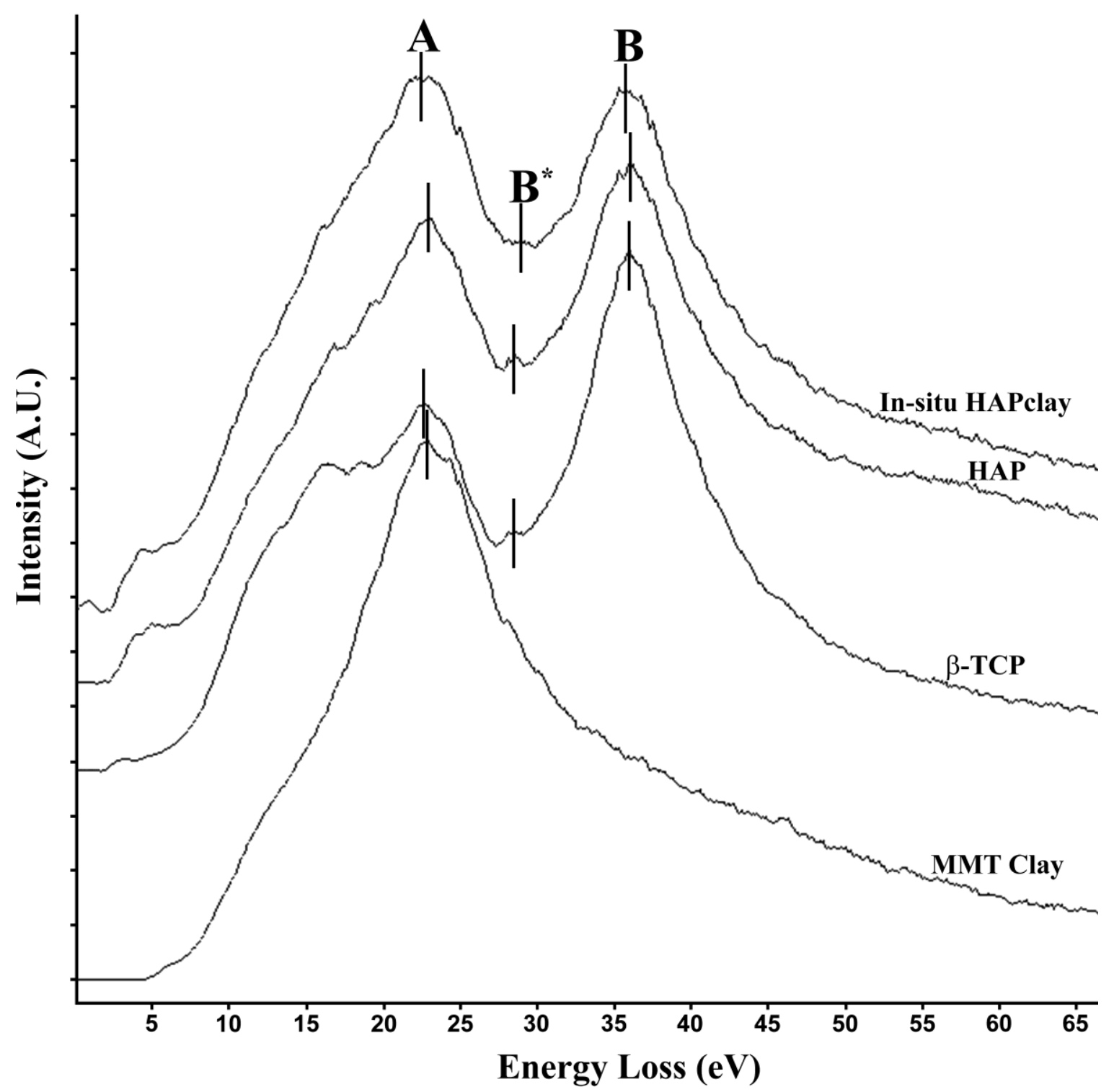


Figure 4

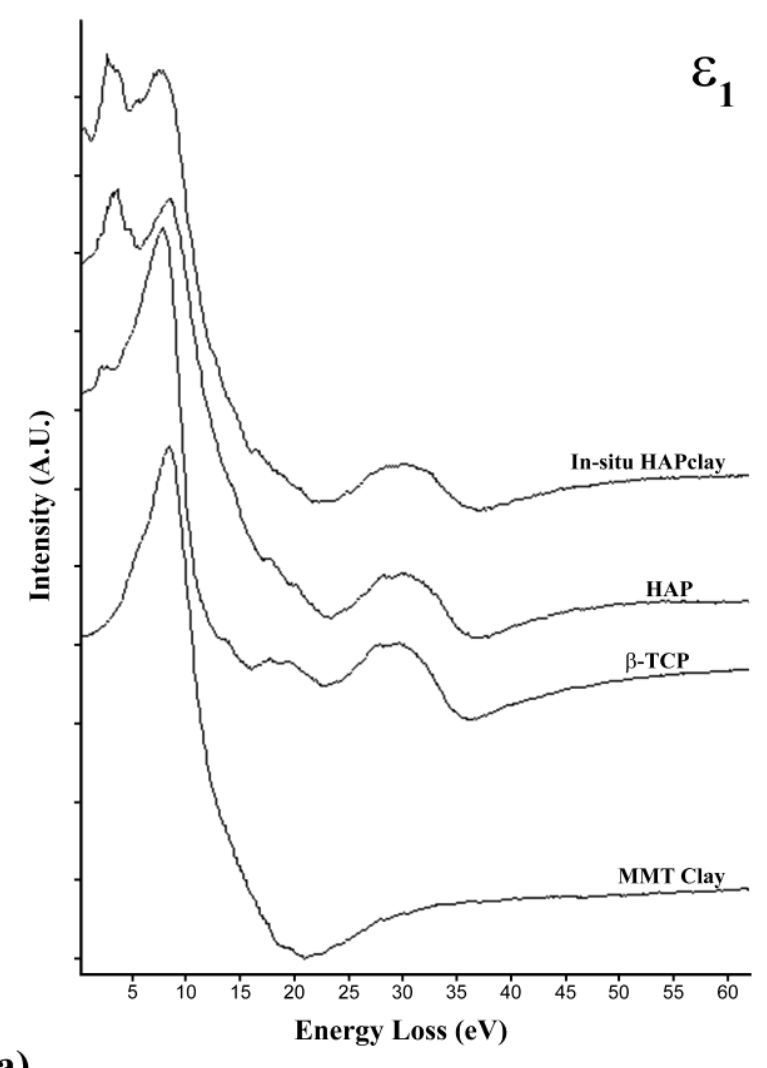

(a)

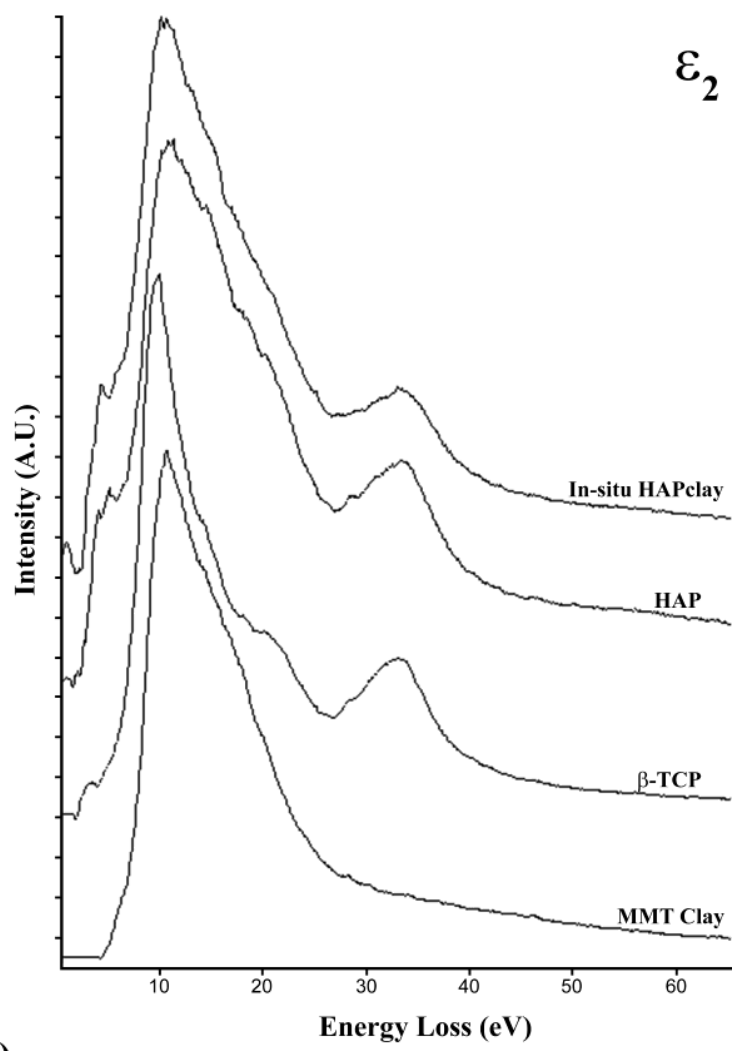

(b) 
Figure 5

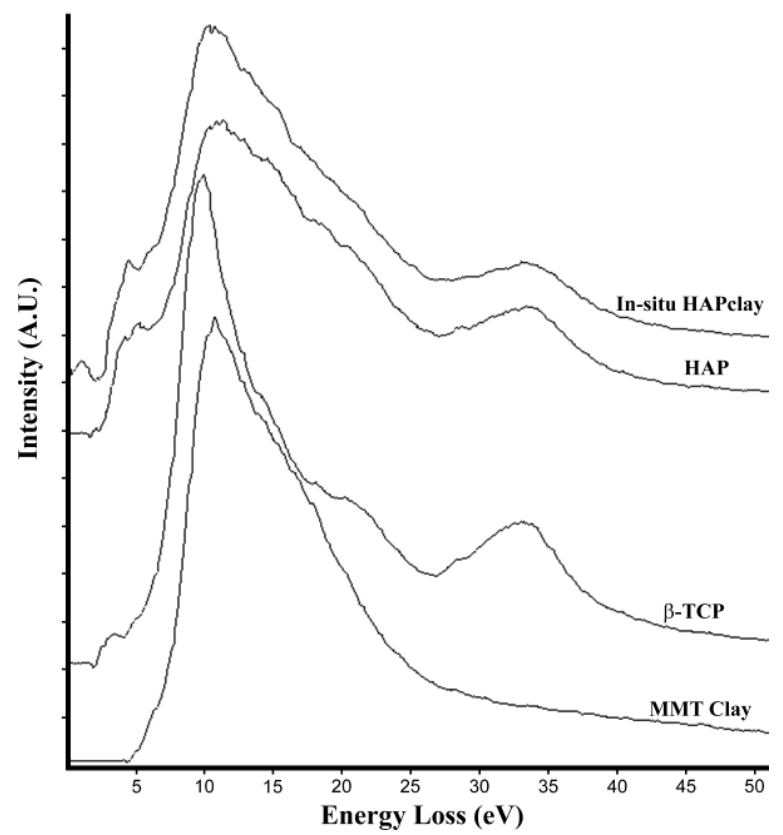


Figure 6

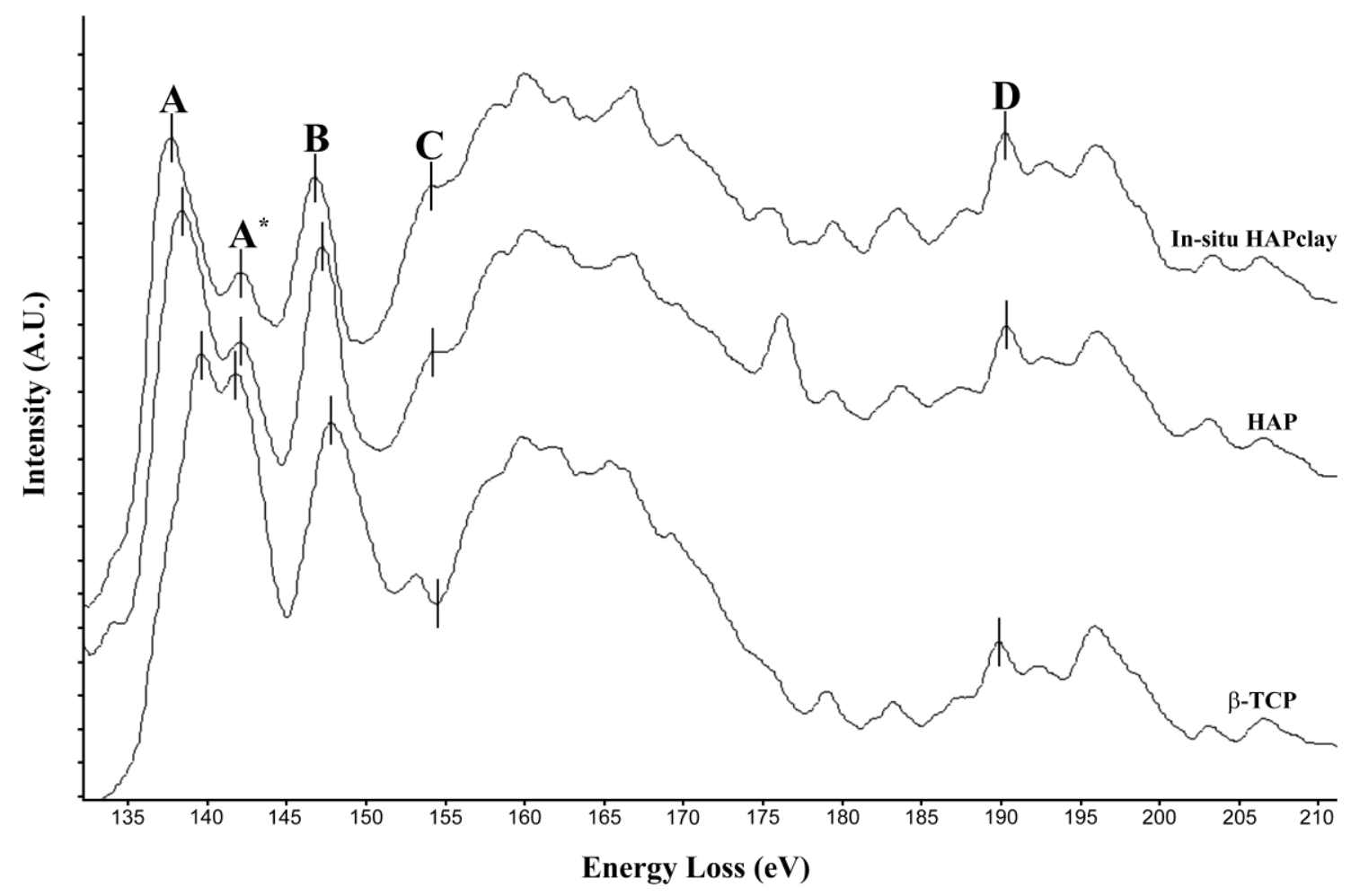


Table 1. Energy Loss Function Peak Positions (eV)

\begin{tabular}{|l|l|l|l|l|}
\cline { 2 - 5 } \multicolumn{1}{c|}{} & $\begin{array}{l}\text { In-situ } \\
\text { HAPclay }\end{array}$ & HAP & $\beta-$ TCP & $\begin{array}{l}\text { Modified } \\
\text { MMT Clay }\end{array}$ \\
\hline Peak A & 22.7 & 22.9 & 22.7 & 23.0 \\
\hline Peak B & 29.8 & 28.7 & 28.5 & -- \\
\hline Peak B & 35.7 & 36.4 & 36.2 & -- \\
\hline
\end{tabular}

Table 2. Energy Loss Function A:B Ratio and Peak Shift(eV)

\begin{tabular}{|c|c|c|c|}
\hline & $\begin{array}{l}\text { In-situ } \\
\text { HAPclay }\end{array}$ & HAP & $\beta$-ТCP \\
\hline A:B Ratio & $\sim 1$ & $<1$ & $<1$ \\
\hline Peak B & -0.7 & 0 & -0.2 \\
\hline Peak B ${ }^{*}$ & +1.3 & +0.2 & 0 \\
\hline
\end{tabular}


Table 3. Justifications of $\varepsilon_{2}$ Transitions (eV)

\begin{tabular}{|c|c|c|c|c|}
\hline $\begin{array}{l}\text { In-situ } \\
\text { HAPclay }\end{array}$ & HAP & $\beta-\mathrm{TCP}$ & $\begin{array}{l}\text { Mod. } \\
\text { MMT } \\
\text { Clay }\end{array}$ & Assignment of Electron Transition Origin \\
\hline 1.0 & -- & -- & -- & \\
\hline-- & -- & 3.0 & -- & \\
\hline 4.1 & 3.9 & -- & -- & \\
\hline 5.8 & -- & -- & -- & \\
\hline 9.7 & 9.8 & 9.4 & 10.5 & $\begin{array}{l}\text { Exciton state (Alexandrov, Y et al 1989; } \\
\text { Philipp H, 1971) }\end{array}$ \\
\hline 13.3 & 12.5 & -- & -- & $\begin{array}{l}\text { Valence band at }-12.9 \mathrm{eV} \text { to conduction } \\
\text { band edge (Philipp H, 1972; Ibach H and } \\
\text { Rowe J, 1974) }\end{array}$ \\
\hline 15.2 & 14.8 & 14.8 & -- & $\begin{array}{l}\text { Valence band to exciton (Philipp H, 1972; } \\
\text { Griscom D, 1977) }\end{array}$ \\
\hline 17.6 & -- & -- & 17.4 & $\begin{array}{l}\text { Valence band to exciton (Philipp H, 1972; } \\
\text { Griscom D, 1977) }\end{array}$ \\
\hline-- & 18.5 & 18.3 & -- & \\
\hline 19.5 & -- & -- & -- & \\
\hline 21.1 & 20.7 & 20.5 & -- & $\begin{array}{l}\text { Valence band to exciton (Griscom D, } \\
\text { 1977) }\end{array}$ \\
\hline-- & 22.0 & -- & -- & $\begin{array}{l}\text { Valence band to exciton (Ibach } \mathrm{H} \text { and } \\
\text { Rowe J, 1974; Griscom D, 1977) }\end{array}$ \\
\hline 25.1 & -- & -- & -- & $\begin{array}{l}\text { O 2s, P 3s to exciton (Rotole J, Sherwood } \\
\text { P 1998, 2001; Welsh I, Sherwood P 1992) }\end{array}$ \\
\hline 28.8 & 28.4 & 28.3 & -- & O 2s to exciton (Griscom D, 1977) \\
\hline 30.9 & 30.6 & -- & -- & \\
\hline 33.3 & 33.8 & 33.8 & -- & $\begin{array}{l}\text { O 2s to conduction band (Griscom D, } \\
\text { 1977) }\end{array}$ \\
\hline
\end{tabular}


Table 4. P-L $\mathrm{L}_{2,3}$ Edge Position (eV) and Electronic Transition Assignments

\begin{tabular}{|c|c|c|c|c|}
\hline Peak & $\beta$-ТCP & HAP & $\begin{array}{l}\text { In-situ } \\
\text { HAPclay }\end{array}$ & Transition Origin \\
\hline Peak A & 139.7 & 138.4 & 137.8 & $\begin{array}{l}\text { Transitions to } 3 p \text {-like anti-bonding state, allowable to } \\
\text { dipole forbidden } 3 p \text { orbitals due to mixed characters } \\
\text { from oxygen (Harp G, et al 1990) }\end{array}$ \\
\hline Peak A* & 141.8 & 142.0 & 142.1 & $\begin{array}{l}\text { Phosphorus } 2 p \text { to Ca } 3 d \text { empty orbitals (Sutherland D, } \\
\text { et al } 1993 \text {; Kruse J, et al 2009) }\end{array}$ \\
\hline Peak B & 147.8 & 147.2 & 146.9 & $\begin{array}{l}\text { d-state shape resonance peak ( } \mathrm{Li} \mathrm{D} \text {, et al 1993; Kruse } \\
\mathrm{J} \text {, et al 2009)) }\end{array}$ \\
\hline Peak C & 153.1 & 154.3 & 154.1 & $\begin{array}{l}\text { Atomic cross section maximum for the } 2 p \text { level (Yeh } \\
\text { J, Landau I, 1985; Sutherland D, et al 1993) }\end{array}$ \\
\hline Peak D & 189.9 & 190.4 & 190.3 & $\mathrm{PL}_{1}$ peak (Ahn C, Krivanek O, 1983) \\
\hline
\end{tabular}

Table 5. P-L2,3 Energy Shifts

\begin{tabular}{|l|c|c|c|c|}
\cline { 2 - 5 } \multicolumn{1}{c|}{} & \multicolumn{2}{c|}{$\begin{array}{c}\text { In-situ HAPclay } \\
\text { shift (eV) }\end{array}$} & \multicolumn{2}{c|}{ HAP shift (eV) } \\
\cline { 2 - 5 } \multicolumn{1}{c|}{} & $\beta-\mathrm{TCP}$ & HAP & $\beta-T C P$ & In-situ HAPclay \\
\hline Peak A & -1.9 & -0.6 & -1.3 & +0.6 \\
\hline Peak A* & +0.3 & +0.1 & +0.2 & -0.1 \\
\hline Peak B & -0.9 & -0.3 & -0.6 & +0.3 \\
\hline Peak C & +0.1 & -0.2 & +1.2 & +0.2 \\
\hline Peak D & +0.4 & -0.1 & +0.5 & +0.1 \\
\hline
\end{tabular}

